\title{
The distribution and correlates of self-rated health in elderly Chinese: the China Kadoorie Biobank study
}

Xingyue Song ${ }^{1}$, Jing $\mathrm{Wu}^{1}$, Canqing $\mathrm{Yu}^{2}$, Wenhong Dong ${ }^{1}$, Jun Lv², Yu Guo ${ }^{3}$, Zheng Bian ${ }^{3}$, Ling Yang ${ }^{4}$, Yiping Chen ${ }^{4}$, Zhengming Chen ${ }^{4}, \mathrm{An} \mathrm{Pan}^{1 *}$, Liming $\mathrm{Li}^{2,3^{*}}$ and on behalf of the China Kadoorie Biobank Collaborative Group

\begin{abstract}
Background: Self-rated health (SRH) have been widely used as a valid indicator of health status at the population and individual level. We aimed to investigate the distribution and correlates of global SRH and age-comparative SRH in elderly Chinese.

Methods: Survey of 57,693 men and 67,089 women aged 60 years and above was conducted in five rural (Gansu, Sichuan, Hunan, Henan, Zhejiang) and five urban areas (Heilongjiang, Shandong, Jiangsu, Guangxi, Hainan) in China between 2004 and 2008. Logistic regression models were used to calculate the relations of different factors with global SRH and age-comparative SRH.

Results: Among the participants, 38.33\% reported their global SRH as good or excellent while $61.67 \%$ as fair or poor, and $17.70 \%$ reported better age-comparative SRH while $17.99 \%$ as worse. In the multivariate model, compared to women, men tended to report a good global SRH and better age-comparative SRH, urban residents tend to report good global SRH and better age-comparative SRH. The socioeconomic and health behavior factors that were associated with good global SRH and better age-comparative SRH (with varying strengths of association) included: high educational level, high household income, house ownership, quitting smoking by own choices, occasional and current alcohol drinking, overweight, and high physical activity level. The factors that were associated with poor global SRH and worse age-comparative SRH included: quitting smoking by illness, former drinking, underweight, and weight lost $\geq 2.5 \mathrm{~kg}$ in the previous year.

Conclusions: We found a moderate level of good global SRH and a low level of better age-comparative SRH among elderly Chinese. We identified a number of demographic, socioeconomic and health behavior factors that were related to SRH measures. Our study emphasizes the importance of incorporating both global and agecomparative SRH measures in future studies, and considering gender inequalities and urban/rural disparity, as well as socioeconomic status and health behaviors as important modifiers of health.
\end{abstract}

Keywords: Distribution, Correlates, Self-rated health, Chinese population

\footnotetext{
* Correspondence: panan@hust.edu.cn; Imleeph@vip.163.com

${ }^{1}$ Department of Epidemiology and Biostatistics, and Ministry of Education Key Laboratory of Environment and Health, and State Key Laboratory of

Environmental Health (Incubating), School of Public Health, Tongji Medical College, Huazhong University of Science and Technology, 13 Hangkong Rd, Wuhan 430030, China

${ }^{2}$ Department of Epidemiology and Biostatistics, School of Public Health, Peking University Health Science Center, 38 Xueyuan Rd, Beijing 100191, China

Full list of author information is available at the end of the article
}

(c) The Author(s). 2019 Open Access This article is distributed under the terms of the Creative Commons Attribution 4.0 International License (http://creativecommons.org/licenses/by/4.0/), which permits unrestricted use, distribution, and reproduction in any medium, provided you give appropriate credit to the original author(s) and the source, provide a link to the Creative Commons license, and indicate if changes were made. The Creative Commons Public Domain Dedication waiver (http://creativecommons.org/publicdomain/zero/1.0/) applies to the data made available in this article, unless otherwise stated. 


\section{Background}

Self-rated health (SRH) is an indicator widely used to reflect a person's general health condition and to measure health inequalities in epidemiology and public health survey. Selfhealth assessment is a cognitive process [1], and it is usually measured by a single question "How would you rate your health in general?", with a three- to five-point scale from "excellent" to "very poor" [2], or by asking about "health status compared to other people of your age", with three potential choices of "better", "the same" and "worse" [3]. SRH is a valid predictor of mortality, disability, physical performance and frailty in older adults, and the validity has been tested in populations from Europe, Northern and South America, Oceania and most areas of Asia, including China [2, 4-6]. $\mathrm{SRH}$ has also been recommended by the World Health Organization as an indicator for health monitoring [7].

As the most populous country in the world, population aging has become a serious social problem in China. It is estimated that the proportion of Chinese people aged 60 and above will reach $28 \%$ by 2040 [8], and represent $65 \%$ of Chinese health burden [9]. Understanding the health status and correlates of SRH in older people is important for providing appropriate health services. Previous studies on the correlates of SRH has been extensively studied in Western populations, and reported that SRH can be affected by a range of demographic (e.g., age, gender) [10, 11], socioeconomic (e.g., marital status, education level, income) [10-12], lifestyle (e.g., smoking, alcohol, physical activity and body mass index) $[10,11,13,14]$, comorbidities and psychological factors [10, 12, 15]. Nevertheless, comprehensive assessments of the correlates of SRH among Chinese populations are lacking. Only a few studies are available, but the sample size of most studies was small and cannot represent a vast and populous country like China [16-18]. Furthermore, most existing studies focus on general global SRH, and very few studies have included the comparative (such as age-referential) SRH measure and it remains unclear whether the correlates of global and age-comparative SRH are the same or different. A previous study examined the correlates of agecomparative SRH in the Swedish population, but did not include global SRH [19]. Only one study in Finland has used both global and age-comparative SRH measures, but only investigated the relationship of age and function ability with SRH [20]. Health conditions and disease status are major correlates of SRH, as shown in many studies [3, $12,18]$ and also our previous analysis [21], but more emphases should be given to the upstream social determinants of health, such as gender inequality, urban/rural disparity, socioeconomic status, and health behaviors [22].

Therefore, using data from the China Kadoorie Biobank (CKB), we aimed to investigate the distribution and correlates of good global SRH and better agecomparative $\mathrm{SRH}$ in elderly Chinese.

\section{Methods}

\section{Study population}

The CKB study is a general population-based prospective cohort study of over 0.5 million participants from $10 \mathrm{di}-$ verse areas across China. The detailed study design, sampling strategy and characteristics of the study participants are previously reported [23]. Briefly, a total of 512,891 participants aged 30-79 years old were recruited from five rural (Tianshui, Gansu Province; Pengzhou, Sichuan Province; Liuyang, Hunan Province; Huixian, Henan Province; Tongxiang, Zhejiang Province) and five urban areas (Harbin, Heilongjiang Province; Qingdao, Shandong Province; Suzhou, Jiangsu Province; Liuzhou, Guangxi Province; Haikou, Hainan Province) between 2004 and 2008. The study areas were selected considering a range of disease patterns and risk factors, quality of death and disease registries, and local commitment and capacity. In each study area, the subdistrict or township administrative region was designated as the investigation unit, and the potentially eligible participants selected for the study within each region were identified through official residential records, and invitation letters (with study information leaflets) were delivered door-to-door by local community leaders or health workers, following extensive publicity campaigns. As a prerequisite for participating, all participants were asked to bring their unique national identity (ID) cards to the assessment centre set up in the local community. The current study focused on the correlates of SRH among people aged 60 years and above, thus we excluded the participants younger than 60 years $(n=388,108)$.

In the baseline survey, detailed information including general demographic characteristics, socioeconomic status, lifestyle factors, mental health and history of chronic diseases was collected by trained interviewers using a laptopbased direct data-entry system. Body height, weight, hip and waist circumference and blood pressures were measured by trained technicians.

All the participants had complete data on the variables necessary for the current analysis except for 1 participants with missing values of body mass index (BMI). Therefore, this participant was excluded and a total of 124,782 participants were available for the final analysis. The study was approved by the ethical review committee of the Chinese Center for Disease Control and Prevention (Beijing, China) and the Oxford Tropical Research Ethics Committee, University of Oxford (UK). Written informed consent forms were obtained from all participants.

\section{Data collection}

In this study, SRH status was assessed using the following two questions: 1) How is your current general health status: excellent, good, fair, or poor? 2) How is your current health status compared with someone of your age: better, about the same, worse, or don't know? We treated the first 
question as global SRH and the second one as agecomparative SRH. The global SRH was categorized into two categories in the analyses: good (excellent, good) and poor (fair, poor). We excluded the participants who answer "don't know" ( $n=5271,4.22 \%)$ and "about the same" $(n=$ $74,983,60.09 \%$ ) for the second question when analyzing the correlates of age-comparative SRH.

Demographic and socioeconomic factors included age, gender, study location (urban/rural), marital status (married, widowed, separated/divorced, never married), education level (no formal education, primary, middle or high school, college/ university or higher), annual household income $(<1450,1450$ 2899, 2890-5072, $\geq 5073$ US dollars, and 1 dollar approximately equals to 6.9 Yuan), and house ownership (yes or no).

Lifestyle factors included cigarette smoking (never, former, occasionally and current smoker), alcohol consumption (never, former, occasionally, and current drinker) and weight change during the past year (unchanged, gained $\geq 2.5 \mathrm{~kg}$ and lost $\geq 2.5 \mathrm{~kg}$ ). For former smokers, the main reason for cessation (already ill or stopped by choice) was also asked. The physical activity level was calculated by adding up metabolic equivalent tasks (METs) for daily work or leisure activities, and was classified into sex-specific quartiles. BMI was calculated as weight in kilograms divided by the square of height in meters and categorized according to the Chinese classification [24]: BMI $<18.5$ as underweight, $18.5 \leq \mathrm{BMI}<24.0$ as normal weight, $24.0 \leq$ $\mathrm{BMI}<28.0$ as overweight and BMI $\geq 28.0$ as obesity.

Seven types of comorbidities including cardiometabolic diseases (diabetes, hypertension, coronary heart disease, stroke, rheumatic heart disease), respiratory diseases (tuberculosis, emphysema/bronchitis, asthma), digestive diseases (cirrhosis/chronic hepatitis, peptic ulcer, gallstone/gallbladder disease), musculoskeletal diseases (fracture, rheumatic arthritis), mental diseases (psychiatric disorders, neurasthenia, depression and generalized anxiety disorder), cancer and other diseases (kidney disease, head injury) were self-reported or measured at baseline. We have previously evaluated the relations of various comorbidities with global and age-comparative SRH measures in this cohort and the details were introduced elsewhere [21].

\section{Statistical analyses}

Baseline characteristics by gender were presented by unadjusted means with standard deviations (SD) for continuous variables and unadjusted proportions for categorical variables, and compared using ANOVA and Chi-square tests for continuous and categorical variables, respectively. Logistic regression models were used to calculate the associations between different factors and SRH measures. The variables were adjusted in the following steps: model 1 included age, gender and study location (10 areas); model 2 additionally included social and economic indicators (marital status, education level, household income, and homeownership); model 3 further added health behaviors (smoking, alcohol, physical activity, BMI and weight change) and baseline comorbidities. We also conducted a sensitivity analysis of including participants answering "about the same" for the agecomparative $\mathrm{SRH}$, and multinomial logistic regression models were used for the three-category outcome.

Stratified analyses were performed according to gender and residential area (urban and rural). Tests for interaction were conducted by adding interaction terms of the study factors and the stratifying variable in the final model. All analyses were performed using SAS 9.3 (SAS Institute Inc.), and a two-sided $P$ value $<0.05$ was considered as statistical significance.

\section{Results}

\section{Characteristics of the participants}

The baseline characteristics stratified by gender are presented in Table 1 . Of the 124,782 participants, $46.24 \%$ were men and $53.76 \%$ were women. The mean age was 66.50 years for men and 66.10 years for women. Compared with women, men were more likely to be married, to have a higher education level, to be current smokers and current drinkers. A total of $38.33 \%$ reported their global SRH as "excellent" or "good" (42.15\% in men and $35.06 \%$ in women, referred to "good" thereafter), and $17.70 \%$ reported better agecomparative SRH (20.40\% in men and $15.37 \%$ in women).

\section{Factors associated with good global SRH}

Table 2 shows the relations of demographic, socioeconomic factors and health behaviors with good global SRH. In the final model, the odds of reporting good global SRH was significantly higher in men and urban residents. Other factors that were associated with good global SRH included high educational level, high household income, house ownership, quitting smoking by own choices, occasional and current alcohol drinking, high level of physical activity and overweight. On the other hand, quitting smoking because of illness, former alcohol drinking, underweight and significant weight loss in the past year were associated with a lower odds of reporting good global SRH. Age and marital status were also related to global SRH, but the effect estimates were modest.

Similar findings were obtained in the stratified analyses by gender (Table 3), although the magnitude of the association varied between different strata. High educational level, high household income, occasional and current alcohol drinking, overweight and high level of physical activity was associated with good global SRH in both subgroups. The positive association between educational level and global SRH was stronger in men. Former alcohol drinking was associated with poor global SRH among men, while showed no significant association among women. Significant weight gain in the past year was associated with good global SRH among men, while showed no significant association among women. In 
Table 1 Characteristics of the study participants at baseline in the China Kadoorie Biobank study, 2004-2008

\begin{tabular}{|c|c|c|c|c|}
\hline Variables & Total $(n=124782)$ & Male $(n=57693)$ & Female $(n=67089)$ & $P$ Valuet \\
\hline Global SRH status & & & & $<0.001$ \\
\hline Excellent & $17537(14.05)$ & $9201(15.95)$ & $8336(12.43)$ & \\
\hline Good & $30300(24.28)$ & $15115(26.20)$ & $15185(22.63)$ & \\
\hline Fair & $60181(48.24)$ & $26778(46.41)$ & 33403 (49.79) & \\
\hline Poor & $16764(13.43)$ & $6599(11.44)$ & $10165(15.15)$ & \\
\hline \multicolumn{5}{|l|}{ Age-comparative SRH status } \\
\hline Better & $22083(17.70)$ & $11772(20.40)$ & $10311(15.37)$ & \\
\hline About the same & $74983(60.09)$ & $35088(60.83)$ & 39895 (59.47) & \\
\hline Worse & 22445 (17.99) & $8765(15.19)$ & $13680(20.39)$ & \\
\hline Don't know & $5271(4.22)$ & $2068(3.58)$ & $3203(4.77)$ & \\
\hline Age, mean (SD), yr & $66.29(4.37)$ & $66.50(4.40)$ & $66.10(4.33)$ & $<0.001$ \\
\hline Rural residence & $63771(51.11)$ & $31615(54.80)$ & 32156 (47.93) & $<0.001$ \\
\hline Married & 98555 (78.98) & $50533(87.59)$ & $48022(71.58)$ & $<0.001$ \\
\hline Educational level & & & & $<0.001$ \\
\hline No formal school & 40476 (32.44) & $10192(17.67)$ & $30284(45.15)$ & \\
\hline Primary school & 48444 (38.82) & $25745(44.62)$ & 22699 (33.83) & \\
\hline Middle or high school & $29153(23.36)$ & $17000(29.47)$ & $12153(18.11)$ & \\
\hline College or university & $6709(5.38)$ & $4756(8.24)$ & $1953(2.91)$ & \\
\hline Annual household income, US Dollar ${ }^{b}$ & & & & $<0.001$ \\
\hline $0-1449$ & $45480(36.45)$ & $19696(34.15)$ & $25784(38.43)$ & \\
\hline $1450-2899$ & $35072(28.11)$ & $16145(27.98)$ & $18927(28.21)$ & \\
\hline $2890-5072$ & $26052(20.88)$ & $12590(21.82)$ & $13462(20.07)$ & \\
\hline$\geq 5073$ & $18178(14.56)$ & $9262(16.05)$ & $8916(13.29)$ & \\
\hline House ownership (Yes) & $50160(40.20)$ & $23973(41.55)$ & 26187 (39.03) & $<0.001$ \\
\hline Smoking status & & & & $<0.001$ \\
\hline Never smoker & $70871(56.80)$ & $10853(18.81)$ & $60018(89.46)$ & \\
\hline Former smoker and quit by choices & $6396(5.12)$ & $5585(9.68)$ & $811(1.21)$ & \\
\hline Former smoker and quit by illness & $7894(6.33)$ & $6873(11.91)$ & $1021(1.52)$ & \\
\hline Occasional smoker & $7171(5.75)$ & $5563(9.64)$ & $1608(2.40)$ & \\
\hline Current smoker & $32450(26.00)$ & $28819(49.96)$ & $3631(5.41)$ & \\
\hline Alcohol status & & & & $<0.001$ \\
\hline Never drinker & $64717(51.86)$ & $16939(29.36)$ & $47778(71.22)$ & \\
\hline Former drinker & $4662(3.74)$ & $4079(7.07)$ & $583(0.87)$ & \\
\hline Occasional drinker & $32130(25.75)$ & $16075(27.86)$ & 16055 (23.93) & \\
\hline Current drinker & $23273(18.65)$ & $20600(35.71)$ & $2673(3.98)$ & \\
\hline BMI, mean (SD) & $23.43(3.62)$ & $22.94(3.32)$ & $23.85(3.81)$ & $<0.001$ \\
\hline Physical activity (MET-h/day), mean (SD) & $13.39(10.37)$ & $13.33(11.90)$ & $13.44(8.85)$ & 0.08 \\
\hline Weight change in the past year & & & & $<0.001$ \\
\hline Lost $\geq 2.5 \mathrm{~kg}$ & $13278(10.64)$ & $5895(10.22)$ & $7383(11.00)$ & \\
\hline Change $\pm 2.5 \mathrm{~kg}$ & $102698(82.30)$ & $47887(83.00)$ & $54811(81.70)$ & \\
\hline Gained $\geq 2.5 \mathrm{~kg}$ & $8806(7.06)$ & 3911 (6.78) & $4895(7.30)$ & \\
\hline Cardiometabolic diseases ${ }^{c}$ (Yes) & $76650(61.43)$ & $34440(59.70)$ & $42210(62.92)$ & $<0.001$ \\
\hline Respiratory diseases ${ }^{c}$ (Yes) & $9968(7.99)$ & $5768(10.00)$ & $4200(6.26)$ & $<0.001$ \\
\hline Musculoskeletal diseases ${ }^{c}$ (Yes) & $13469(10.79)$ & $5355(9.28)$ & $8114(12.09)$ & $<0.001$ \\
\hline
\end{tabular}


Table 1 Characteristics of the study participants at baseline in the China Kadoorie Biobank study, 2004-2008 (Continued)

\begin{tabular}{|c|c|c|c|c|}
\hline Variables & Total $(n=124782)$ & Male $(n=57693)$ & Female $(n=67089)$ & $P$ Valuet \\
\hline Mental diseases ${ }^{\complement}($ Yes $)$ & $2822(2.26)$ & $829(1.44)$ & $1993(2.97)$ & $<0.001$ \\
\hline Digestives diseases ${ }^{c}$ (Yes) & $16201(12.98)$ & $6799(11.78)$ & $9402(14.01)$ & $<0.001$ \\
\hline Cancer (Yes) & $1078(0.86)$ & $528(0.92)$ & $550(0.82)$ & $<0.001$ \\
\hline Other diseases ${ }^{c}$ (Yes) & $13085(2.55)$ & $550(2.90)$ & $6993(2.31)$ & 0.04 \\
\hline
\end{tabular}

Abbreviations: BMI Body mass index, MET Metabolic equivalent task, SD Standard deviations, SRH Self-rated health

${ }^{\mathrm{a}}$ Data are presented as frequency (percentage) unless otherwise indicated

${ }^{\mathrm{b}}$ At the exchange rate as of December 2018, 1 US dollar approximately equals to 6.9 RMB Yuan

${ }^{c}$ Cardiometabolic diseases include diabetes, hypertension, coronary heart disease, stroke and rheumatic heart disease; Respiratory diseases include tuberculosis, emphysema/bronchitis and asthma; Digestive diseases include cirrhosis/chronic hepatitis, peptic ulcer and gallstone/gallbladder disease; Musculoskeletal diseases include fracture and rheumatic arthritis; Mental diseases include psychiatric disorders, neurasthenia, depression and generalized anxiety disorder; Other diseases include kidney disease and head injury

† Two-sided $P$ values were derived from ANOVA for continuous variables and Chi-square test for categorical variables

the stratified analysis by residential area (Table 3), high educational level, high household income, occasional and current alcohol drinking, and high level of physical activity was associated with good global SRH in both subgroups. The positive association between educational level, household income and global SRH was stronger among rural residents, while the association between physical activity and global SRH was stronger among urban residents. Significant weight gain in the past year were associated with good global SRH among rural residents, but not among urban residents. Although significant interactions were found for some other variables probably because of large sample size, the effect estimates were not substantially different across strata.

\section{Factors associated with better age-comparative SRH}

Table 4 shows the results for better age-comparative SRH in the total study population. In the final model, the odds of reporting better age-comparative SRH was significantly higher in men, urban residents and older individuals. Other factors that were associated with better age-comparative SRH included being windowed, high educational level, high household income, house ownership, quitting smoking by own choices, occasional and current alcohol drinking, high level of physical activity and overweight. The factors that associated with worse age-comparative SRH included quitting smoking because of illness, former alcohol drinking, underweight and significant weight loss.

In the stratified analyses by gender (Table 5), older age, high household income, occasional and current alcohol drinking, high level of physical activity, overweight was associated with better age-comparative SRH in both subgroups. The positive association between age, household income, overweight and age-comparative SRH was stronger in men. Former alcohol drinking was associated with worse age-comparative SRH in men, but not in women. In the stratified analysis by residential area (Table 5), high educational level, high household income, occasional and current alcohol drinking, high level of physical activity and overweight was associated with better age-comparative SRH in both subgroups. The positive association between educational level, household income and age-comparative SRH was stronger among rural residents, while the association between physical activity and age-comparative SRH was stronger among urban residents. Significant weight gain in the past year were associated with better agecomparative SRH among rural residents, while show opposite association among urban residents. Although significant interactions were found for some other variables, the effect estimates were not substantially different.

In the sensitivity analysis of including participants who answered "about the same" for the age-comparative SRH, the results did not change materially (Table 6 in Appendix).

\section{Discussion}

In this large population-based study, we found a moderate level of good global SRH (38.33\%) and a low level of better age-comparative SRH (17.70\%) among elderly Chinese. In general, men and urban residents were more likely to report good/better SRH compared to women and rural residents. People with high socioeconomic status (education level, household income and house ownership) and health behaviors (physical activity, low to moderate alcohol consumption, quitting smoking by own choices) were more likely to report good/better SRH, while people with underweight or significant weight loss in the past year were more likely to report poor/worse SRH. Age was positively associated with agecomparative SRH, indicating a survival advantage.

Some previous studies have reported large variations in the level of good/excellent global SRH in Chinese populations, ranging from 25.2 to $47 \%$ in different studies $[6,16$, $18,25,26]$. As noted, the global SRH is highly influenced by the characteristics of the study population (age, gender composition, urban/rural, health status etc.) and large variations are expected due to the different selection criteria in various studies. Therefore, direct comparison of our study results to those previous reports in the Chinese populations may not be meaningful. Most prior studies were restricted to subpopulations in one or two certain areas or institutions, and the sample size ranged from 411 to 12,583 . Our study is possibly the largest population-based study from 
Table 2 Multivariate adjusted correlates of good global self-rated health status in the China Kadoorie Biobank study, 2004-2008

\begin{tabular}{|c|c|c|c|c|c|c|}
\hline \multirow[t]{2}{*}{ Variables } & \multicolumn{2}{|c|}{ Model 1} & \multicolumn{2}{|c|}{ Model 2} & \multicolumn{2}{|c|}{ Model $3^{b}$} \\
\hline & OR & $95 \% \mathrm{Cl}$ & $\mathrm{OR}$ & $95 \% \mathrm{Cl}$ & $\mathrm{OR}$ & $95 \% \mathrm{Cl}$ \\
\hline \multicolumn{7}{|l|}{ Demographic factors } \\
\hline \multicolumn{7}{|l|}{ Age (ref. $=60-64 \mathrm{yr}$ ) } \\
\hline $65-69 y r$ & 0.91 & $0.88,0.93$ & 0.93 & $0.90,0.95$ & 1.02 & $0.99,1.05$ \\
\hline$\geq 70 \mathrm{yr}$ & 0.84 & $0.81,0.86$ & 0.88 & $0.85,0.91$ & 1.05 & $1.01,1.08$ \\
\hline Gender (ref. $=$ female) & 1.41 & $1.37,1.44$ & 1.35 & $1.31,1.38$ & 1.26 & $1.22,1.31$ \\
\hline Residential area (ref. $=$ Rural) & 1.23 & $1.20,1.25$ & 1.20 & $1.17,1.23$ & 1.28 & $1.24,1.32$ \\
\hline \multicolumn{7}{|l|}{ Socioeconomic factors } \\
\hline \multicolumn{7}{|l|}{ Marital status (ref. $=$ Married) } \\
\hline windowed & & & 1.04 & $1.01,1.07$ & 1.06 & $1.02,1.09$ \\
\hline Separated/divorced/never married & & & 0.84 & $0.75,0.94$ & 0.86 & $0.77,0.97$ \\
\hline \multicolumn{7}{|c|}{ Educational level (ref. = No formal school) } \\
\hline Primary school & & & 1.04 & $1.01,1.08$ & 1.05 & $1.01,1.08$ \\
\hline Middle or high school & & & 1.13 & $1.09,1.17$ & 1.17 & $1.12,1.22$ \\
\hline College or university & & & 1.32 & $1.24,1.41$ & 1.41 & $1.32,1.50$ \\
\hline \multicolumn{7}{|c|}{ Annual household income, US Dollar (ref. =0-1449) } \\
\hline 1450-2899 & & & 1.10 & $1.07,1.14$ & 1.11 & $1.07,1.14$ \\
\hline $2890-5072$ & & & 1.15 & $1.10,1.19$ & 1.14 & $1.10,1.19$ \\
\hline$\geq 5073$ & & & 1.29 & $1.23,1.34$ & 1.29 & $1.23,1.35$ \\
\hline House ownership (ref. = No) & & & 1.16 & $1.13,1.20$ & 1.16 & $1.12,1.19$ \\
\hline \multicolumn{7}{|l|}{ Health behaviors } \\
\hline \multicolumn{7}{|l|}{ Smoking status (ref. = Never smoker) } \\
\hline Former smoker and quit by choices & & & & & 1.14 & $1.08,1.21$ \\
\hline Former smoker and quit by illness & & & & & 0.66 & $0.62,0.70$ \\
\hline Occasional smoker & & & & & 0.93 & $0.88,0.98$ \\
\hline Current smoker & & & & & 0.99 & $0.95,1.03$ \\
\hline \multicolumn{7}{|l|}{ Alcohol status (ref. = Never drinker) } \\
\hline Former drinker & & & & & 0.80 & $0.75,0.86$ \\
\hline Occasional drinker & & & & & 1.24 & $1.20,1.28$ \\
\hline Current drinker & & & & & 1.41 & $1.36,1.47$ \\
\hline \multicolumn{7}{|l|}{ Physical activity (MET-h/day) (ref. = Q1) } \\
\hline Q2 & & & & & 1.33 & $1.29,1.37$ \\
\hline Q3 & & & & & 1.41 & $1.36,1.47$ \\
\hline Q4 & & & & & 1.55 & $1.48,1.63$ \\
\hline \multicolumn{7}{|l|}{ BMI category (ref. = Normal weight) } \\
\hline Underweight & & & & & 0.70 & $0.67,0.74$ \\
\hline Overweight & & & & & 1.10 & $1.07,1.13$ \\
\hline Obesity & & & & & 0.96 & $0.92,1.00$ \\
\hline \multicolumn{7}{|c|}{ Weight change in the past year (ref. = Change $\pm 2.5 \mathrm{~kg}$ ) } \\
\hline Lost $\geq 2.5 \mathrm{~kg}$ & & & & & 0.75 & $0.72,0.78$ \\
\hline Gained $\geq 2.5 \mathrm{~kg}$ & & & & & 1.08 & $1.03,1.13$ \\
\hline
\end{tabular}

Abbreviations: BMI Body mass index, Cl Confidence intervals, MET Metabolic equivalent task, OR Odds ratio

${ }^{a}$ For residential area, adjusted for all other variables shown in the table. For other factors, adjusted for 10 study location and all other variables shown in the table ${ }^{b}$ Model 3: adjusted for all other variables plus cardiometabolic diseases, respiratory diseases, musculoskeletal diseases, mental diseases, digestive diseases, cancer and other diseases 
Table 3 Multivariate adjusted correlates of good global self-rated health by residential area and gender in the China Kadoorie Biobank study, 2004-2008

\begin{tabular}{|c|c|c|c|c|c|c|c|c|c|c|}
\hline \multirow[t]{2}{*}{ Variables } & \multicolumn{2}{|l|}{ Male } & \multicolumn{2}{|c|}{ Female } & \multirow[t]{2}{*}{$P$ for interaction } & \multicolumn{2}{|l|}{ Rural } & \multicolumn{2}{|c|}{ Urban } & \multirow{2}{*}{$\begin{array}{l}P \text { for } \\
\text { interaction }\end{array}$} \\
\hline & $\mathrm{OR}$ & $95 \% \mathrm{Cl}$ & $\mathrm{OR}$ & $95 \% \mathrm{Cl}$ & & $\mathrm{OR}$ & $95 \% \mathrm{Cl}$ & OR & $95 \% \mathrm{Cl}$ & \\
\hline \multicolumn{11}{|l|}{ Demographic factors } \\
\hline Age (ref. = 60-64 yr) & & & & & 0.24 & & & & & 0.10 \\
\hline $65-69 y r$ & 1.04 & $0.99,1.08$ & 1.00 & $0.96,1.04$ & & 0.99 & $0.95,1.03$ & 1.06 & $1.02,1.11$ & \\
\hline$\geq 70 \mathrm{yr}$ & 1.10 & $1.04,1.15$ & 1.00 & $0.95,1.05$ & & 1.00 & $0.95,1.05$ & 1.10 & $1.05,1.15$ & \\
\hline Gender (ref. = Female) & & & & & & 1.21 & $1.15,1.28$ & 1.35 & $1.28,1.43$ & 0.54 \\
\hline Residential area (ref. $=$ Rural) & 1.26 & $1.21,1.32$ & 1.29 & $1.24,1.34$ & 0.54 & & & & & \\
\hline \multicolumn{11}{|l|}{ Socioeconomic factors } \\
\hline Marital status (ref. $=$ Married) & & & & & 0.03 & & & & & 0.003 \\
\hline windowed & 1.01 & $0.95,1.07$ & 1.08 & $1.04,1.12$ & & 0.99 & $0.95,1.04$ & 1.12 & $1.06,1.17$ & \\
\hline Separated/divorced/never married & 0.82 & $0.72,0.94$ & 1.08 & $0.85,1.36$ & & 0.82 & $0.71,0.95$ & 0.97 & $0.81,1.18$ & \\
\hline Educational level (ref. $=$ No formal school) & & & & & $<0.001$ & & & & & $<0.001$ \\
\hline Primary School & 1.01 & $0.96,1.07$ & 1.09 & $1.04,1.14$ & & 1.09 & $1.04,1.14$ & 0.99 & $0.94,1.04$ & \\
\hline Middle or high School & 1.17 & $1.10,1.25$ & 1.14 & $1.08,1.21$ & & 1.25 & $1.17,1.32$ & 1.11 & $1.05,1.18$ & \\
\hline College or university & 1.40 & $1.28,1.53$ & 1.32 & $1.18,1.47$ & & 1.71 & $1.35,2.17$ & 1.37 & $1.26,1.48$ & \\
\hline \multicolumn{5}{|c|}{ Annual household income, US Dollar (ref. =0-1449) } & 0.66 & & & & & $<0.001$ \\
\hline $1450-2899$ & 1.13 & $1.08,1.19$ & 1.08 & $1.04,1.14$ & & 1.16 & $1.11,1.21$ & 1.03 & $0.98,1.09$ & \\
\hline $2890-5072$ & 1.17 & $1.11,1.24$ & 1.12 & $1.06,1.18$ & & 1.22 & $1.15,1.29$ & 1.09 & $1.03,1.15$ & \\
\hline$\geq 5073$ & 1.36 & $1.27,1.45$ & 1.25 & $1.17,1.33$ & & 1.59 & $1.48,1.70$ & 1.13 & $1.06,1.20$ & \\
\hline House ownership (ref. = No) & 1.15 & $1.10,1.20$ & 1.17 & $1.12,1.22$ & $<0.001$ & 1.14 & $1.08,1.20$ & 1.16 & $1.11,1.20$ & $<0.001$ \\
\hline \multicolumn{11}{|l|}{ Health behaviors } \\
\hline Smoking status (ref. = Never smoker) & & & & & 0.08 & & & & & $<0.001$ \\
\hline Former smoker and quit by choices & 1.13 & $1.05,1.21$ & 1.10 & $0.94,1.28$ & & 1.08 & $0.98,1.18$ & 1.18 & $1.09,1.28$ & \\
\hline Former smoker and quit by illness & 0.64 & $0.60,0.69$ & 0.74 & $0.63,0.86$ & & 0.65 & $0.60,0.71$ & 0.67 & $0.61,0.72$ & \\
\hline Occasional smoker & 0.90 & $0.84,0.97$ & 0.94 & $0.83,1.05$ & & 0.94 & $0.87,1.02$ & 0.91 & $0.83,0.99$ & \\
\hline Current smoker & 0.97 & $0.93,1.02$ & 1.03 & $0.95,1.11$ & & 0.99 & $0.94,1.05$ & 0.99 & $0.93,1.05$ & \\
\hline Alcohol status (ref. = Never drinker) & & & & & 0.001 & & & & & $<0.001$ \\
\hline Former drinker & 0.81 & $0.74,0.88$ & 0.96 & $0.79,1.17$ & & 0.86 & $0.79,0.95$ & 0.71 & $0.63,0.79$ & \\
\hline Occasional drinker & 1.25 & $1.19,1.31$ & 1.23 & $1.18,1.29$ & & 1.29 & $1.23,1.35$ & 1.20 & $1.15,1.26$ & \\
\hline Current drinker & 1.42 & $1.35,1.48$ & 1.56 & $1.43,1.70$ & & 1.43 & $1.36,1.51$ & 1.37 & $1.30,1.45$ & \\
\hline Physical activity (MET-h/day) (ref. = Q1) & & & & & 0.07 & & & & & $<0.001$ \\
\hline Q2 & 1.35 & $1.29,1.41$ & 1.34 & $1.29,1.39$ & & 1.32 & $1.26,1.37$ & 1.35 & $1.30,1.41$ & \\
\hline Q3 & 1.42 & $1.34,1.51$ & 1.40 & $1.33,1.48$ & & 1.25 & $1.19,1.32$ & 1.72 & $1.61,1.83$ & \\
\hline Q4 & 1.71 & $1.60,1.84$ & 1.42 & $1.32,1.52$ & & 1.35 & $1.27,1.43$ & 2.17 & $1.96,2.39$ & \\
\hline BMI category (ref. $=$ Normal weight) & & & & & 0.18 & & & & & 0.13 \\
\hline Underweight & 0.67 & $0.63,0.72$ & 0.72 & $0.67,0.78$ & & 0.74 & $0.69,0.78$ & 0.64 & $0.58,0.71$ & \\
\hline Overweight & 1.11 & $1.06,1.16$ & 1.09 & $1.05,1.14$ & & 1.07 & $1.03,1.12$ & 1.13 & $1.09,1.18$ & \\
\hline Obesity & 0.99 & $0.92,1.07$ & 0.94 & $0.89,0.99$ & & 0.90 & $0.84,0.97$ & 1.01 & $0.95,1.06$ & \\
\hline \multicolumn{5}{|c|}{ Weight change in the past year (ref. $=$ Change $\pm 2.5 \mathrm{~kg}$ ) } & 0.10 & & & & & $<0.001$ \\
\hline Lost $\geq 2.5 \mathrm{~kg}$ & 0.72 & $0.67,0.76$ & 0.77 & $0.73,0.81$ & & 0.69 & $0.65,0.73$ & 0.79 & $0.75,0.84$ & \\
\hline Gained $\geq 2.5 \mathrm{~kg}$ & 1.11 & $1.04,1.19$ & 1.05 & $0.98,1.12$ & & 1.15 & $1.07,1.23$ & 1.02 & $0.96,1.09$ & \\
\hline
\end{tabular}


Table 4 Multivariate adjusted correlates of better age-comparative self-rated health status in the China Kadoorie Biobank study, $2004-2008^{a}$

\begin{tabular}{|c|c|c|c|c|c|c|}
\hline \multirow[t]{2}{*}{ Variables } & \multicolumn{2}{|c|}{ Model 1} & \multicolumn{2}{|c|}{ Model 2} & \multicolumn{2}{|c|}{ Model $3^{b}$} \\
\hline & OR & $95 \% \mathrm{Cl}$ & OR & $95 \% \mathrm{Cl}$ & $\mathrm{OR}$ & $95 \% \mathrm{Cl}$ \\
\hline \multicolumn{7}{|l|}{ Demographic factors } \\
\hline \multicolumn{7}{|l|}{ Age (ref. $=60-64 \mathrm{yr})$} \\
\hline $65-69 y r$ & 0.92 & $0.88,0.97$ & 0.99 & $0.95,1.04$ & 1.21 & $1.15,1.27$ \\
\hline$\geq 70 \mathrm{yr}$ & 0.97 & $0.92,1.02$ & 1.12 & $1.06,1.18$ & 1.63 & $1.54,1.73$ \\
\hline Gender (ref. = Female) & 1.90 & $1.83,1.98$ & 1.65 & $1.58,1.73$ & 1.43 & $1.33,1.53$ \\
\hline Residential area (ref. $=$ Rural) & 2.19 & $2.11,2.28$ & 1.70 & $1.63,1.78$ & 1.93 & $1.84,2.03$ \\
\hline \multicolumn{7}{|l|}{ Socioeconomic factors } \\
\hline \multicolumn{7}{|l|}{ Marital status (ref. = Married) } \\
\hline windowed & & & 1.15 & $1.09,1.21$ & 1.20 & $1.13,1.26$ \\
\hline Separated/divorced/never married & & & 0.85 & $0.70,1.03$ & 0.95 & $0.77,1.16$ \\
\hline \multicolumn{7}{|l|}{ Educational level (ref. = No formal school) } \\
\hline Primary school & & & 1.24 & $1.18,1.31$ & 1.27 & $1.20,1.35$ \\
\hline Middle or high school & & & 1.70 & $1.59,1.82$ & 1.84 & $1.72,1.98$ \\
\hline College or university & & & 2.36 & $2.12,2.64$ & 2.76 & $2.45,3.12$ \\
\hline \multicolumn{7}{|c|}{ Annual household income, US Dollar (ref. =0-1449) } \\
\hline $1450-2899$ & & & 1.20 & $1.14,1.27$ & 1.21 & $1.14,1.28$ \\
\hline $2890-5072$ & & & 1.34 & $1.25,1.43$ & 1.35 & $1.26,1.45$ \\
\hline$\geq 5073$ & & & 1.56 & $1.44,1.68$ & 1.58 & $1.45,1.71$ \\
\hline House ownership (ref. = No) & & & 1.28 & $1.28,1.35$ & 1.30 & $1.23,1.37$ \\
\hline \multicolumn{7}{|l|}{ Health behaviors } \\
\hline \multicolumn{7}{|l|}{ Smoking status (ref. $=$ Never smoker) } \\
\hline Former smoker and quit by choices & & & & & 1.32 & $1.18,1.48$ \\
\hline Former smoker and quit by illness & & & & & 0.50 & $0.45,0.55$ \\
\hline Occasional smoker & & & & & 1.01 & $0.92,1.12$ \\
\hline Current smoker & & & & & 1.05 & $0.98,1.13$ \\
\hline \multicolumn{7}{|l|}{ Alcohol status (ref. = Never drinker) } \\
\hline Former drinker & & & & & 0.67 & $0.59,0.75$ \\
\hline Occasional drinker & & & & & 1.62 & $1.53,1.71$ \\
\hline Current drinker & & & & & 2.13 & $1.99,2.28$ \\
\hline \multicolumn{7}{|l|}{ Physical activity (MET-h/day) (ref.=Q1) } \\
\hline Q2 & & & & & 1.78 & $1.69,1.88$ \\
\hline Q3 & & & & & 2.36 & $2.20,2.52$ \\
\hline Q4 & & & & & 2.77 & $2.53,3.04$ \\
\hline \multicolumn{7}{|l|}{ BMl category (ref. = Normal weight) } \\
\hline Underweight & & & & & 0.49 & $0.45,0.54$ \\
\hline Overweight & & & & & 1.30 & $1.24,1.37$ \\
\hline Obesity & & & & & 0.99 & $0.92,1.07$ \\
\hline \multicolumn{7}{|c|}{ Weight change in the past year (ref. $=$ Change $\pm 2.5 \mathrm{~kg}$ ) } \\
\hline Lost $\geq 2.5 \mathrm{~kg}$ & & & & & 0.57 & $0.54,0.61$ \\
\hline Gained $\geq 2.5 \mathrm{~kg}$ & & & & & 1.04 & $0.96,1.12$ \\
\hline
\end{tabular}

Abbreviations: $B M I$ Body mass index, $C l$ Confidence intervals, MET Metabolic equivalent task, OR Odds ratio

"Participants answering "don't know" $(n=5271)$ and "about the same" $(n=74,983)$ for the age-comparative self-rated health status question were excluded from the analysis, leaving 44,528 participants in this analysis. For residential area, adjusted for all other variables shown in the table. For other factors, adjusted for 10 study location and all other variables shown in the table

${ }^{b}$ Model 3: adjusted for all other variables plus cardiometabolic diseases, respiratory diseases, musculoskeletal diseases, mental diseases, digestive diseases, cancer and other diseases 
10 diverse regions of China focusing on correlates of SRH. Our prevalence estimate (38.33\% reporting good/excellent global SRH) was comparable to a previous study among 1433 participants aged 60 years and above in one urban and two rural areas of Beijing and Shanghai (38.1\%) [6].

Most participants assessed their age-comparative SRH as "about the same" (60.09\%), $17.70 \%$ as "better" and $17.99 \%$ as "worse". Similar levels of age-comparative SRH were found in a study among 62,824 residents aged $\geq 65$ years in Hong Kong (72.9\% as "about the same" and $25.3 \%$ as "better") [5]. However, a study in Finland found a level of similar age-comparative SRH of $28 \%$ and a level of better age-comparative SRH of $42 \%$ [27]. The Chinese "doctrine of the mean" advocates modesty which may make people more likely to rate their age-comparative SRH as "about the same" or "similar". Again, the selection criteria of the study population may also have a big influence.

Among the demographic variables, we found that older people were more likely to report better age-comparative SRH but similar global SRH, the results of age-comparative SRH were consistent with previous studies, but the results of global SRH were not entirely consistent with previous studies $[3,19,20]$. When reporting global SRH, people may compare their current health status with that of their younger ages or with other younger people, but in the context of agecomparative SRH, they compared their health status with someone of similar ages. Survival bias is a possible explanation for better age-comparative SRH related to old age because those elderly people who participated in the study could have generally good health status compared to those who could not participate (e.g., because of premature death or severe diseases). Previous studies indicated that elderly people tended to overestimate their own heath or underestimate the health of others of their ages, and there may be a deterioration of judgment with increasing age or a healthy survivor effect $[3,20]$. In addition, the perception of health status could be different in old people, the continuous reduction in the level of health expectations allows older people to better adapt to the aging process. We also found that men tended to report a good global SRH and better agecomparative SRH compared with women, which was consistent with most previous studies [12, 19]. Gender differences in SRH may be due to the differences in social status, social stress, family roles, pain tolerance and health expectations between men and women [28]. Men in our study were more likely to have a higher education level, although we have controlled for education level in the models, residual confounding of socioeconomic status is still possible and we could not adequately adjust for other aspects of the socioeconomic inequalities between men and women. In addition, compared with women, men are more tolerant of various physical pains and more optimistic about health, and the gender differences in mental health status (e.g., women reporting higher probability of depression) have been well- established [29]. Urban residents were more likely to report good/better SRH status in our study. The rural residents in our study were mostly engaged in agricultural work, had low social status and low income, and there were dramatic differences in living environment and medical conditions between urban and rural areas. Taken together, our study cannot fully explain the exact reasons for the gender and urban/rural differences in reporting health status, but since gender inequality and urban/rural health disparity are generally upstream determinants of socioeconomic status and health behaviors, our results further emphasize the importance of establishing social policy to achieve greater social and economic equality in society in order to reduce the health inequity.

The relations of marital status with SRH were not consistently reported in the literature $[12,18,19]$, and we found that the associations were generally null or modest. Consistent with previous studies [16-19], we found that people with higher educational level and household income levels were more likely to report better global and age-comparative SRH. People with higher level of education and income may have better living conditions, better recognition of healthy lifestyles, and greater ability to withstand health risks and control their own health. Therefore, our results echo the recommendations from the WHO Commission on Social Determinants of Health that more actions are needed to tackle the health disparity by focusing on social determinants [22].

As for health behaviors, we found that healthy lifestyles (physical activity and low to moderate alcohol consumption) were associated with both good global SRH and better agecomparative $\mathrm{SRH}$, although the associations were generally stronger for age-comparative SRH. We did not find significant association with current smoking, while the association with past smoking depended on the reason of quitting smoking. People who quitted smoking because of illness were less likely to report good SRH status, which may reflect the fact that they were in poor physical health status. In our study, people who quitted smoking by their own choices were more likely to report good SRH status, which may be because those individuals paid more attention to their lifestyles and health status. Current drinking was significantly associated with better SRH in our study, particularly for age-comparative SRH, which is consistent with another study in China [18]. The reason may be that regular drinker have formed a suitable amount of drinking over the lifetime, and this lifestyle can bring social benefits, pleasure and relieve fatigue. It should be noted that most of the current drinkers drank low-to-moderate amount of alcohol, which has been shown to be part of healthy lifestyles and associated with better health outcomes, including healthy ageing [30]. On the contrast, former alcohol drinkers were less likely to report good SRH, and it is possible that many people stopped drinking because of physical health conditions. Consistent with previous studies [18, 19, 31], we also found that high levels of physical activity were associated with good SRH status. Regular physical activity can help improve 
Table 5 Multivariate adjusted correlates of better age-comparative self-rated health status by residential area and gender in the China Kadoorie Biobank study, 2004-2008

\begin{tabular}{|c|c|c|c|c|c|c|c|c|c|c|}
\hline \multirow[t]{2}{*}{ Variables } & \multicolumn{2}{|l|}{ Male } & \multicolumn{2}{|c|}{ Female } & \multirow[t]{2}{*}{$P$ for interaction } & \multicolumn{2}{|l|}{ Rural } & \multicolumn{2}{|c|}{ Urban } & \multirow[t]{2}{*}{$P$ for interaction } \\
\hline & $\mathrm{OR}$ & $95 \% \mathrm{Cl}$ & $\mathrm{OR}$ & $95 \% \mathrm{Cl}$ & & $\mathrm{OR}$ & $95 \% \mathrm{Cl}$ & $\mathrm{OR}$ & $95 \% \mathrm{Cl}$ & \\
\hline \multicolumn{11}{|l|}{ Demographic factors } \\
\hline Age (ref. = 60-64 yr) & & & & & 0.03 & & & & & 0.64 \\
\hline $65-69 y r$ & 1.25 & $1.16,1.36$ & 1.18 & $1.10,1.26$ & & 1.17 & $1.09,1.27$ & 1.25 & $1.16,1.35$ & \\
\hline$\geq 70 \mathrm{yr}$ & 1.88 & $1.72,2.06$ & 1.45 & $1.34,1.57$ & & 1.60 & $1.46,1.74$ & 1.67 & $1.54,1.81$ & \\
\hline Gender (ref. = Female) & & & & & & 1.35 & $1.22,1.48$ & 1.55 & $1.40,1.70$ & 0.02 \\
\hline Residential area (ref. = Rural) & 1.69 & $1.56,1.83$ & 2.13 & $2.00,2.28$ & 0.02 & & & & & \\
\hline \multicolumn{11}{|l|}{ Socioeconomic factors } \\
\hline Marital status (ref. $=$ Married) & & & & & 0.05 & & & & & 0.009 \\
\hline windowed & 1.07 & $0.96,1.19$ & 1.24 & $1.16,1.32$ & & 1.09 & $1.00,1.17$ & 1.30 & $1.20,1.42$ & \\
\hline Separated/divorced/never married & 0.93 & $0.72,1.18$ & 1.05 & $0.72,1.55$ & & 0.79 & $0.60,1.05$ & 1.21 & $0.89,1.65$ & \\
\hline \multicolumn{3}{|l|}{ Educational level (ref.= No formal school) } & & & 0.01 & & & & & $<0.001$ \\
\hline Primary School & 1.27 & $1.16,1.40$ & 1.29 & $1.20,1.39$ & & 1.32 & $1.22,1.42$ & 1.21 & $1.11,1.32$ & \\
\hline Middle or high School & 1.98 & $1.77,2.22$ & 1.68 & $1.53,1.85$ & & 2.06 & $1.84,2.29$ & 1.70 & $1.54,1.88$ & \\
\hline College or university & 2.75 & $2.34,3.25$ & 2.77 & $2.30,3.34$ & & 3.23 & $2.15,4.84$ & 2.60 & $2.26,2.99$ & \\
\hline \multicolumn{3}{|c|}{ Annual household income, US Dollar (ref. $=0-1449$ ) } & & & 0.02 & & & & & $<0.001$ \\
\hline $1450-2899$ & 1.30 & $1.19,1.42$ & 1.15 & $1.07,1.24$ & & 1.22 & $1.13,1.33$ & 1.18 & $1.08,1.29$ & \\
\hline $2890-5072$ & 1.50 & $1.34,1.67$ & 1.28 & $1.17,1.41$ & & 1.52 & $1.34,1.73$ & 1.32 & $1.20,1.45$ & \\
\hline$\geq 5073$ & 1.91 & $1.68,2.17$ & 1.38 & $1.24,1.55$ & & 1.98 & $1.68,2.34$ & 1.50 & $1.35,1.67$ & \\
\hline House ownership (ref. = No) & 1.25 & $1.15,1.36$ & 1.33 & $1.24,1.43$ & 0.03 & 1.24 & $1.14,1.36$ & 1.31 & $1.22,1.40$ & $<0.001$ \\
\hline \multicolumn{11}{|l|}{ Health behaviors } \\
\hline \multicolumn{3}{|l|}{ Smoking status (ref. = Never smoker) } & & & $<0.001$ & & & & & 0.43 \\
\hline Former smoker and quit by choices & 1.32 & $1.15,1.51$ & 1.06 & $0.84,1.35$ & & 1.40 & $1.17,1.67$ & 1.27 & $1.10,1.47$ & \\
\hline Former smoker and quit by illness & 0.45 & $0.40,0.51$ & 0.64 & $0.52,0.80$ & & 0.52 & $0.45,0.61$ & 0.48 & $0.42,0.55$ & \\
\hline Occasional smoker & 0.98 & $0.86,1.12$ & 0.90 & $0.75,1.07$ & & 1.07 & $0.94,1.22$ & 0.96 & $0.82,1.12$ & \\
\hline Current smoker & 1.01 & $0.92,1.11$ & 1.04 & $0.93,1.18$ & & 1.09 & $0.99,1.20$ & 1.01 & $0.91,1.12$ & \\
\hline \multicolumn{3}{|l|}{ Alcohol status (ref. = Never drinker) } & & & $<0.001$ & & & & & $<0.001$ \\
\hline Former drinker & 0.63 & $0.55,0.72$ & 1.21 & $0.92,1.60$ & & 0.82 & $0.70,0.96$ & 0.51 & $0.42,0.61$ & \\
\hline Occasional drinker & 1.73 & $1.58,1.89$ & 1.53 & $1.43,1.65$ & & 1.64 & $1.52,1.78$ & 1.62 & $1.50,1.75$ & \\
\hline Current drinker & 2.19 & $2.01,2.40$ & 2.50 & $2.16,2.89$ & & 2.27 & $2.05,2.50$ & 2.00 & $1.81,2.21$ & \\
\hline \multicolumn{3}{|l|}{ Physical activity (MET-h/day) (ref.=Q1) } & & & 0.06 & & & & & $<0.001$ \\
\hline Q2 & 1.87 & $1.72,2.03$ & 1.75 & $1.63,1.87$ & & 1.69 & $1.56,1.83$ & 1.85 & $1.72,1.98$ & \\
\hline Q3 & 2.38 & $2.14,2.65$ & 2.29 & $2.09,2.50$ & & 2.12 & $1.93,2.32$ & 2.72 & $2.44,3.03$ & \\
\hline Q4 & 3.33 & $2.89,3.83$ & 2.38 & $2.10,2.70$ & & 2.29 & $2.04,2.58$ & 4.06 & $3.42,4.81$ & \\
\hline \multicolumn{3}{|l|}{ BMI category (ref. = Normal weight) } & & & 0.003 & & & & & 0.01 \\
\hline Underweight & 0.47 & $0.42,0.54$ & 0.50 & $0.44,0.57$ & & 0.56 & $0.50,0.63$ & 0.40 & $0.34,0.47$ & \\
\hline Overweight & 1.45 & $1.34,1.57$ & 1.20 & $1.12,1.28$ & & 1.19 & $1.10,1.28$ & 1.39 & $1.30,1.49$ & \\
\hline Obesity & 1.14 & $1.00,1.29$ & 0.91 & $0.83,0.99$ & & 0.90 & $0.79,1.02$ & 1.07 & $0.97,1.17$ & \\
\hline \multicolumn{5}{|c|}{ Weight change in the past year (ref. $=$ Change $\pm 2.5 \mathrm{~kg}$ ) } & 0.002 & & & & & $<0.001$ \\
\hline Lost $\geq 2.5 \mathrm{~kg}$ & 0.53 & $0.48,0.59$ & 0.61 & $0.56,0.66$ & & 0.55 & $0.50,0.61$ & 0.59 & $0.54,0.64$ & \\
\hline Gained $\geq 2.5 \mathrm{~kg}$ & 1.15 & $1.02,1.30$ & 0.96 & $0.87,1.06$ & & 1.31 & $1.17,1.48$ & 0.86 & $0.78,0.96$ & \\
\hline
\end{tabular}

Odds ratios $(95 \% \mathrm{Cl})$ were calculated after adjustment of other variables shown in the table plus 10 study locations, cardiometabolic diseases, respiratory diseases, musculoskeletal diseases, mental diseases, digestive diseases, cancer and other diseases, except for the stratified variable in the corresponding stratified analysis

Abbreviations: BMI Body mass index, Cl Confidence intervals, MET Metabolic equivalent task, OR Odds ratio

aparticipants answering "don't know" $(n=5271)$ and "about the same" $(n=74,983)$ for the age-comparative self-rated health status question were excluded from the analysis, leaving 44,528 participants in this analysis 
physical and mental function as well as alleviate the adverse effects of some chronic diseases [32], and physical activity is positively associated with healthy ageing [33].

In our study, underweight was significantly associated with poor global SRH and worse age-comparative SRH, consistent with previous studies [14,34]. On the other hand, overweight was associated with better SRH, particularly for agecomparative SRH, which was not in agreement with previous studies in Caucasians [14, 19, 35]. Many Chinese do not consider overweight/obesity as a disease but as a symbol of wealth [36]. Previous studies in China also found an inverse association between overweight and obesity and depression $[37,38]$, and indicated that overweight/obesity people may be more optimistic about life and health, like a saying: "laughing and growing fat is a blessing". When we further examined the impact of weight change on SRH, we found people who lost more than $2.5 \mathrm{~kg}$ weight in the past year were less likely to report both good global SRH and better age-comparative $\mathrm{SRH}$, which may be due to disease-induced weight loss. Meanwhile, people who gained weight more than $2.5 \mathrm{~kg}$ were more likely to report a good global SRH but show no significant association with better age-comparative SRH.

The relations of most variables with the two SRH measures in different subgroups were in the same direction, but the effect sizes varied substantially. We found that socioeconomic status (education level, household income) and health behaviors (smoking and drinking status) had a stronger influence on global SRH and age-comparative SRH in men. Furthermore, socioeconomic status (educational level and household income) had a stronger influence on global SRH and age-comparative SRH among rural residents, and health behaviors (physical activity) had a stronger influence on global SRH and age-comparative SRH among urban residents. The results further confirm the impact of gender inequality and urban-rural economic differences on health, and emphasize the importance of healthy lifestyle to improve personal health among urban residents.

For the correlates of the two SRH measures, our study further confirmed that older people tended to report better agecomparative SRH. Overall, the relations of different variables with the two SRH measures were in the same direction, but the effect sizes varied substantially. For example, the associations were generally stronger for age-comparative SRH with socioeconomic status (education level and household income), health behaviors (alcohol intake, physical activity, BMI and weight change). Although the exact mechanisms are unclear, our study indicated that future studies should incorporate both global and age-comparative SRH to provide complementary information.

The strength of this study is that we used both global and age-comparative SRH measures as outcomes and the analyses covered a wide range of demographic, socioeconomic, and lifestyle factors. The study was based on a large and diverse population that provided us sufficient power to detect modest associations. However, several limitations should be noted. First, the study participants were not selected to represent the whole China, and the generalizability of the study findings should be cautious despite the large sample size. We did not include people aged 80 years and older, and again the generalizability to much older populations is unclear given that the age group over 80 years comprises large proportion of people with disability and poor health. However, our major findings of gender inequalities, urban/rural health divide, socioeconomic status and health behaviors as major correlates of health should still be valid and have important policy implications for the current Chinese society, and even for other populations undergoing nutrition and health transitions. Second, many variables were self-reported and the findings may be subject to residual confounding and recall bias if people with different SRH levels recalled things differently, although the participants were face-to-face interviewed by well-trained interviewers. Third, because of the large sample size, even modest effect size could be statistically significant, including the interaction tests; therefore, the results should be interpreted cautiously and we only discussed variables that showed relatively strong associations with the outcomes. Fourth, several factors which have been found to be associated with SRH in previous studies, such as functional ability, cognitive levels and social networks [20,34], were not available in this study. Finally, causal relations cannot be determined in a cross-sectional study like ours.

\section{Conclusions}

We found a moderate level of good global SRH and a low level of better age-comparative SRH among elderly Chinese. We identified a number of demographic, socioeconomic and health behaviors that were significantly associated with global and age-comparative $\mathrm{SRH}$, with varying degrees and directions of effect estimates. Therefore, future studies should incorporate both global and age-comparative SRH to provide complementary information on the health status of the individuals and population. The significant differences between men and women, urban and rural residents in reporting SRH and the different correlates of $\mathrm{SRH}$ also indicate the importance of considering gender and urban/rural inequalities as the upstream determinants of health status. In addition, the consistent and strong impact of high socioeconomic status on SRH also provides evidence for reducing health inequity through social policy to achieve greater social and economic equality in society. Finally, our study also highlights the potential of improving personal and population health by modifiable behavior factors that include smoking cessation and regular exercise. 


\section{Appendix}

Table 6 Correlates of better age-comparative self-rated health status in the China Kadoorie Biobank study, 2004-2008: multinomial logistic regression analysis ${ }^{a}$

\begin{tabular}{|c|c|c|c|c|c|}
\hline \multirow[t]{2}{*}{ Variables } & \multirow{2}{*}{$\begin{array}{l}\text { Worse } \\
\text { OR } \\
\end{array}$} & \multicolumn{2}{|c|}{ About the same } & \multicolumn{2}{|c|}{ Better } \\
\hline & & OR & $95 \% \mathrm{Cl}$ & $\mathrm{OR}$ & $95 \% \mathrm{Cl}$ \\
\hline \multicolumn{6}{|l|}{ Demographic factors } \\
\hline \multicolumn{6}{|l|}{ Age (ref. $=60-64$ yr) } \\
\hline $65-69 \mathrm{yr}$ & 1 & 1.06 & $1.02,1.10$ & 1.20 & $1.15,1.26$ \\
\hline$\geq 70 \mathrm{yr}$ & 1 & 1.28 & $1.23,1.34$ & 1.62 & $1.53,1.71$ \\
\hline Sex (ref. $=$ Women $)$ & 1 & 1.23 & $1.17,1.29$ & 1.43 & $1.34,1.52$ \\
\hline Residential area (ref. = Rural) & 1 & 1.08 & $1.04,1.12$ & 1.97 & $1.88,2.07$ \\
\hline \multicolumn{6}{|l|}{ Socioeconomic factors } \\
\hline \multicolumn{6}{|l|}{ Marital status (ref. = Married) } \\
\hline windowed & 1 & 1.05 & $1.00,1.09$ & 1.19 & $1.13,1.25$ \\
\hline Separated/divorced/never married & 1 & 0.85 & $0.74,0.97$ & 0.89 & $0.74,1.08$ \\
\hline \multicolumn{6}{|l|}{ Educational level (ref. = No formal school) } \\
\hline Primary school & 1 & 1.22 & $1.17,1.27$ & 1.27 & $1.20,1.34$ \\
\hline Middle or high school & 1 & 1.33 & $1.26,1.41$ & 1.84 & $1.73,1.97$ \\
\hline College or university & 1 & 1.60 & $1.45,1.77$ & 2.66 & $2.38,2.98$ \\
\hline \multicolumn{6}{|c|}{ Annual household income, US Dollar (ref. $=0-1449$ ) } \\
\hline $1450-2899$ & 1 & 1.17 & $1.12,1.22$ & 1.21 & $1.14,1.27$ \\
\hline $2890-5072$ & 1 & 1.30 & $1.24,1.37$ & 1.36 & $1.28,1.46$ \\
\hline$\geq 5073$ & 1 & 1.32 & $1.25,1.41$ & 1.63 & $1.51,1.76$ \\
\hline House ownership (ref. = No) & 1 & 1.13 & $1.09,1.18$ & 1.32 & $1.26,1.39$ \\
\hline \multicolumn{6}{|l|}{ Health behaviors } \\
\hline \multicolumn{6}{|l|}{ Smoking status (ref. = Never smoker) } \\
\hline Former smoker and quit by choices & 1 & 1.16 & $1.06,1.27$ & 1.29 & $1.17,1.43$ \\
\hline Former smoker and quit by illness & 1 & 0.59 & $0.55,0.64$ & 0.50 & $0.45,0.55$ \\
\hline Occasional smoker & 1 & 0.98 & $0.90,1.06$ & 0.98 & $0.89,1.08$ \\
\hline Current smoker & 1 & 0.98 & $0.93,1.04$ & 1.04 & $0.97,1.11$ \\
\hline \multicolumn{6}{|l|}{ Alcohol status (ref. = Never drinker) } \\
\hline Former drinker & 1 & 0.68 & $0.63,0.73$ & 0.68 & $0.61,0.77$ \\
\hline Occasional drinker & 1 & 1.38 & $1.32,1.44$ & 1.61 & $1.53,1.70$ \\
\hline Current drinker & 1 & 1.60 & $1.52,1.69$ & 2.15 & $2.01,2.30$ \\
\hline \multicolumn{6}{|l|}{ Physical activity (MET-h/day) (ref.= Q1) } \\
\hline Q2 & 1 & 1.48 & $1.42,1.54$ & 1.84 & $1.75,1.93$ \\
\hline Q3 & 1 & 1.78 & $1.69,1.87$ & 2.53 & $2.38,2.70$ \\
\hline Q4 & 1 & 2.10 & $1.96,2.25$ & 2.97 & $2.73,3.24$ \\
\hline \multicolumn{6}{|l|}{ BMI category (ref. $=$ Normal weight) } \\
\hline Underweight & 1 & 0.59 & $0.56,0.63$ & 0.49 & $0.45,0.54$ \\
\hline Overweight & 1 & 1.17 & $1.13,1.22$ & 1.28 & $1.22,1.34$ \\
\hline Obesity & 1 & 1.00 & $0.95,1.05$ & 1.02 & $0.95,1.09$ \\
\hline \multicolumn{6}{|c|}{ Weight change in the past year (ref. $=$ Change $\pm 2.5 \mathrm{~kg}$ ) } \\
\hline Lost $\geq 2.5 \mathrm{~kg}$ & 1 & 0.54 & $0.52,0.57$ & 0.55 & $0.52,0.58$ \\
\hline Gained $\geq 2.5 \mathrm{~kg}$ & 1 & 0.80 & $0.75,0.85$ & 1.00 & $0.93,1.08$ \\
\hline
\end{tabular}

For residential area, adjusted for all other variables plus cardiometabolic diseases, respiratory diseases, musculoskeletal diseases, mental diseases, digestive diseases, cancer and other diseases. For other factors, adjusted for 10 study location, all other variables shown in the table, and plus cardiometabolic diseases, respiratory diseases, musculoskeletal diseases, mental diseases, digestive diseases, cancer and other diseases

Abbreviations: BMI Body mass index, Cl Confidence intervals, MET Metabolic equivalent task, OR Odds ratio

aparticipants answering "don't know" ( $n=5271)$ for the age-comparative self-rated health status question were excluded from the analysis, leaving 119,511 participants in this analysis 


\section{Abbreviations}

BMI: Body mass index; Cl: Confidence intervals; CIDI-SF: Composite International Diagnostic Inventory-short form; CKB: China Kadoorie Biobank; METs: Metabolic equivalent tasks; OR: Odds ratio; SRH: Self-rated health

\section{Publisher's Note}

Springer Nature remains neutral with regard to jurisdictional claims in published maps and institutional affiliations.

\section{Acknowledgements}

The chief acknowledgment is to the participants, the project staff, and the China National Centre for Disease Control and Prevention (CDC) and its regional offices for assisting with the fieldwork. We thank Judith Mackay in Hong Kong; Yu Wang, Gonghuan Yang, Zhengfu Qiang, Lin Feng, Maigeng Zhou, Wenhua Zhao, and Yan Zhang in China CDC; Lingzhi Kong, Xiucheng Yu, and Kun Li in the Chinese Ministry of Health; and Sarah Clark, Martin Radley, Mike Hill, Hongchao Pan, and Jill Boreham in the CTSU, Oxford, for assisting with the design, planning, organization, and conduct of the study.

\section{Authors' contributions}

XYS and AP designed the study analysis. CQY, JL, YG, ZMC and LML are investigators of the CKB study and directed its implementation, including quality assurance and control, dataset management and analytic strategy. ZB, $L Y$, and YPC helped supervise the field activities and designed the study's analytic strategy. XYS, JW, and WHD analyzed the data and prepared the manuscript. All authors have critically commented on and revised the manuscript, and approved the final version. AP and LML are guarantors of the paper.

\section{Funding}

This work was supported by the National Key Research and Development Program of China (2017YFC0907504, 2016YFC0900500, 2016YFC0900501, and 2016YFC0900504) and the National Natural Science Foundation of China (81390540, 81390541, and 81202266). The CKB baseline survey and the first re-survey were supported by a grant from the Kadoorie Charitable Foundation in Hong Kong. The long-term follow-up is supported by grants from the UK Wellcome Trust (202922/Z/16/Z, 088158/Z/09/Z, 104085/Z/14/Z) and Chinese Ministry of Science and Technology (2011BAI09B01). The funders had no role in the study design, data collection, data analysis and interpretation, writing of the report, or the decision to submit the article for publication.

\section{Availability of data and materials}

The data that support the findings of this article are available from the CKB upon reasonable request (http://www.ckbiobank.org/site/Data+Access).

\section{Ethics approval and consent to participate}

The study got approval from the ethical review committees of the Chinese Center for Disease Control and Prevention (Beijing, China) and the Oxford Tropical Research Ethics Committee, University of Oxford (UK). Written informed consent forms were obtained from all participants.

\section{Consent for publication}

Not applicable.

\section{Competing interests}

The authors declare that they have no competing interests.

\section{Author details}

${ }^{1}$ Department of Epidemiology and Biostatistics, and Ministry of Education Key Laboratory of Environment and Health, and State Key Laboratory of Environmental Health (Incubating), School of Public Health, Tongji Medical College, Huazhong University of Science and Technology, 13 Hangkong Rd, Wuhan 430030, China. ${ }^{2}$ Department of Epidemiology and Biostatistics, School of Public Health, Peking University Health Science Center, 38 Xueyuan Rd, Beijing 100191, China. ${ }^{3}$ Chinese Academy of Medical Sciences, Dong Cheng District, Beijing, China. ${ }^{4}$ Clinical Trial Service Unit \& Epidemiological Studies Unit (CTSU), Nuffield Department of Population Health, University of Oxford Oxford, UK.
Received: 18 July 2018 Accepted: 5 June 2019

Published online: 14 June 2019

\section{References}

1. Jylha M. What is self-rated health and why does it predict mortality? Towards a unified conceptual model. Soc Sci Med. 2009;69(3):307-16.

2. DeSalvo KB, Bloser N, Reynolds K, He J, Muntner P. Mortality prediction with a single general self-rated health question. A meta-analysis. J Gen Intern Med. 2006;21:267-75.

3. Bin Li Z, Lam TH, Ho SY, Chan WM, Ho KS, Li MP, et al. Age-versus timecomparative self-rated health in Hong Kong Chinese older adults. Int J Geriatr Psych. 2006;21:729-39.

4. Perez-Zepeda MU, Belanger E, Zunzunegui MV, Phillips S, Ylli A, Guralnik J. Assessing the validity of self-rated health with the short physical performance battery: a cross-sectional analysis of the international mobility in aging study. PLoS One. 2016;11:e0153855.

5. Shen C, Schooling CM, Chan WM, Zhou JX, Johnston JM, Lee SY, et al. Selfrated health and mortality in a prospective Chinese elderly cohort study in Hong Kong. Prev Med. 2014;67:112-8.

6. Feng QS, Zhu HY, Zhen ZH, Gu DA. Self-rated health, interviewer-rated health, and their predictive powers on mortality in old age. J Gerontol BPsychol. 2016;71:538-50.

7. de Bruin A, Picavet HS, Nossikov A. Health interview surveys. Towards international harmonization of methods and instruments. WHO Reg Publ Eur Ser. 1996;58:i-xiii 1-161.

8. World Health Organization. China country assessment report on ageing and health. 2015. http://www.who.int/iris/handle/10665/194271. Accessed 20 Feb 2019.

9. Chatterji S, Kowal P, Mathers C, Naidoo N, Verdes E, Smith JP, Suzman R. The health of aging populations in China and India. Health Aff (Millwood). 2008;27(4):1052-63.

10. Kelleher CC, Whelan J, Daly L, Fitzpatrick P. Socio-demographic, environmental, lifestyle and psychosocial factors predict self rated health in Irish Travellers, a minority nomadic population. Health Place. 2012;18(2):330-8.

11. Stanojevic Jerkovic O, Sauliune S, Sumskas L, Birt CA, Kersnik J. Determinants of self-rated health in elderly populations in urban areas in Slovenia, Lithuania and UK: findings of the EURO-URHIS 2 survey. Eur J Pub Health. 2017;27(suppl 2):74-9.

12. Haseen F, Adhikari R, Soonthorndhada K. Self-assessed health among Thai elderly. BMC Geriatr. 2010;10. https://doi.org/10.1186/1471-2318-10-30.

13. Pisinger $C$, Toft $U$, Aadahl M, Glumer $C$, Jorgensen T. The relationship between lifestyle and self-reported health in a general population: the Inter99 study. Prev Med. 2009:49:418-23.

14. Imai K, Gregg EW, Chen YJ, Zhang P, de Rekeneire N, Williamson DF. The association of BMI with functional status and self-rated health in US adults. Obesity (Silver Spring). 2008;16(2):402-8.

15. Mantzavinis GD, Pappas N, Dimoliatis LDK, loannidis JPA. Multivariate models of self-reported health often neglected essential candidate determinants and methodological issues. J Clin Epidemiol. 2005;58:43643.

16. Liu FF, Zhang CT, Liang YM, Deng QJ, Hang D, Pan YQ, et al. Epidemiology of self-rated health in rural China: a population-based cross-sectional study. Sci Rep-Uk. 2017;7. https://doi.org/10.1038/s41598-017-04381-6.

17. Chen $Y$, While AE, Hicks A. Self-rated health and associated factors among older people living alone in Shanghai. Geriatr Gerontol Int. 2015;15:457-64.

18. Haseli-Mashhadi N, Pan A, Ye XW, Wang J, Qi QB, Liu Y, et al. Self-rated health in middle-aged and elderly Chinese: distribution, determinants and associations with cardio-metabolic risk factors. BMC Public Health. 2009;9. https://doi.org/10.1186/1471-2458-9-368.

19. Waller G, Janlert U, Hamberg K, Forssen A. What does age-comparative selfrated health measure? A cross-sectional study from the northern Sweden MONICA project. Scand J Public Healt. 2016;44:233-9.

20. Vuorisalmi M, Lintonen T, Jylha M. Comparative vs global self-rated health: associations with age and functional ability. Aging Clin Exp Res. 2006;18:211-7.

21. Song $X Y$, Wu J, Yu CQ, Dong WH, Lv J, Guo Y, et al. Association between multiple comorbidities and self-rated health status in middleaged and elderly Chinese: the China Kadoorie biobank study. BMC Public Health. 2018;18. https://doi.org/10.1186/s12889-018-5632-1. 
22. Marmot M, Friel S, Bell R, Houweling TAJ, Taylor S, HIt CSD. Closing the gap in a generation: health equity through action on the social determinants of health. Lancet. 2008;372:1661-9.

23. Chen ZM, Chen JS, Collins R, Guo Y, Peto R, Wu F, et al. China Kadoorie biobank of 0.5 million people: survey methods, baseline characteristics and long-term follow-up. Int J Epidemiol. 2011;40:1652-66.

24. Chen C, Lu FC. Department of disease control ministry of health, People's Rupublic of China. The guidelines for prevention and control of overweight and obesity in Chinese adults. Biomed Environ Sci. 2004;17(Suppl):1-36.

25. Goldman N, Glei DA, Chang MC. The role of clinical risk factors in understanding self-rated health. Ann Epidemiol. 2004;14:49-57.

26. Leung KK, Tang LY, Lue BH. Self-rated health and mortality in Chinese institutional elderly persons. J Clin Epidemiol. 1997;50:1107-16.

27. Vuorisalmi M, Lintonen T, Jylha M. Global self-rated health data from a longitudinal study predicted mortality better than comparative self-rated health in old age. J Clin Epidemiol. 2005;58:680-7.

28. Liu X, Fu X, Liu B, Li H, Zhou L. Analysis on the influencing factors of selfrated health among the elderly by using multilevel model. Chinses J Dis Control Prev. 2018;22(2):173-7 [in Chinese].

29. Seedat S, Scott KM, Angermeyer MC, Berglund P, Bromet EJ, Brugha TS, et al. Cross-National Associations between gender and mental disorders in the World Health Organization world mental health surveys. Arch Gen Psychiat. 2009;66(7):785-95.

30. Sun Q, Townsend MK, Okereke Ol, Rimm EB, Hu FB, Stampfer MJ, et al. Alcohol consumption at midlife and successful ageing in women: a prospective cohort analysis in the Nurses' health study. PLoS Med. 2011;8(9): e1001090

31. Harrington J, Perry IJ, Lutomski J, Fitzgerald AP, Shiely F, McGee H, et al. Living longer and feeling better: healthy lifestyle, self-rated health, obesity and depression in Ireland. Eur J Pub Health. 2010;20:91-5.

32. McPhee JS, French DP, Jackson D, Nazroo J, Pendleton N, Degens H. Physical activity in older age: perspectives for healthy ageing and frailty. Biogerontology. 2016;17(3):567-80.

33. Daskalopoulou C, Stubbs B, Kralj C, Koukounari A, Prince M, Prina AM. Physical activity and healthy ageing: a systematic review and meta-analysis of longitudinal cohort studies. Ageing Res Rev. 2017;38:6-17.

34. Molarius A, Berglund K, Eriksson C, Lambe M, Nordstrom E, Eriksson HG, et al. Socioeconomic conditions, lifestyle factors, and self-rated health among men and women in Sweden. Eur J Pub Health. 2007;17:125-33.

35. Aguilar-Palacio I, Gil-Lacruz Al, Sanchez-Recio R, Rabanaque MJ. Self-rated health in Europe and its determinants: does generation matter? Int J Public Health. 2018;63(2):223-32.

36. Li ZB, Ho SY, Chan WM, Ho KS, Li MP, Leung GM, et al. Obesity and depressive symptoms in Chinese elderly. Int J Geriatr Psychiatry. 2004; 19(1):68-74.

37. Ho RC, Niti M, Kua EH, Ng TP. Body mass index, waist circumference, waisthip ratio and depressive symptoms in Chinese elderly: a population-based study. Int J Geriatr Psychiatry. 2008;23:401-8.

38. Zhang L, Liu K, Li H, Li D, Chen Z, Zhang LL, et al. Relationship between body mass index and depressive symptoms: the "fat and jolly" hypothesis for the middle-aged and elderly in China. BMC Public Health. 2016;16:1201. https://doi.org/10.1186/s12889-016-3864-5.

\section{Ready to submit your research? Choose BMC and benefit from:}

- fast, convenient online submission

- thorough peer review by experienced researchers in your field

- rapid publication on acceptance

- support for research data, including large and complex data types

- gold Open Access which fosters wider collaboration and increased citations

- maximum visibility for your research: over $100 \mathrm{M}$ website views per year

At $\mathrm{BMC}$, research is always in progress.

Learn more biomedcentral.com/submissions 